\title{
El suicidio
}

y otros comportamientos

autodestructivos en jóvenes universitarios

de Colombia y Puerto Rico:

acciones, interacciones

y significaciones

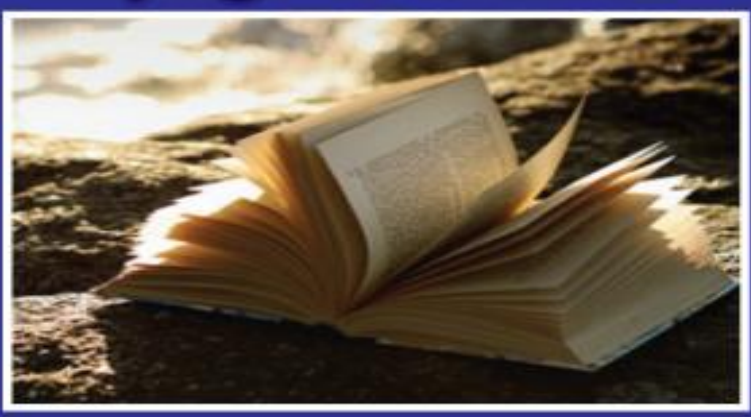

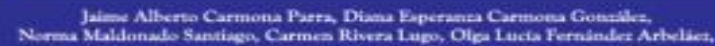

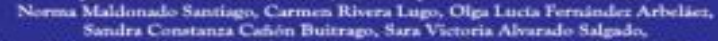

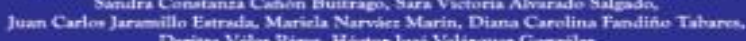

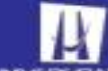

MANIZALES

CAPÍTULO 8. La prevención del suicidio y otros comportamientos autodestructivos en jóvenes universitarios: acciones, interacciones y significaciones.

\section{Modelo de citación}

Carmona Parra, J. A., Alvarado Salgado, S. V., Narváez Marín, M., Cañón Buitrago, S. C. y Carmona González, D. E. (2017). La prevención del suicidio y otros comportamientos autodestructivos en jóvenes universitarios: acciones, interacciones y significaciones. En J.A., Carmona, et al. (Comp.). El suicidio y otros comportamientos autodestructivos en jóvenes universitarios en Colombia y Puerto Rico: acciones interacciones y significaciones. (pp. 161178). Manizales: Universidad de Manizales. 


\title{
8. Suicidio y otros comportamientos autodestructivos en jóvenes universitarios de Colombia y Puerto Rico: análisis comparativo
}

\author{
Jaime Alberto Carmona Parra \\ Sara Victoria Alvarado Salgado \\ Mariela Narváez Marín \\ Sandra Constanza Cañón Buitrago \\ Diana Esperanza Carmona González
}

En las líneas siguientes se aportan algunas orientaciones para la prevención del suicidio en el escenario universitario, partiendo de los resultados de una investigación realizada simultáneamente en Colombia y Puerto Rico en la que participaron 192 estudiantes de ambos países que cumplían con uno de los siguientes criterios: haber realizado un intento de suicidio en el último año, haber presentado ideaciones suicidas en el último año, haber tenido conocimiento del suicidio consumado de un compañero de estudio cercano en el último año o haber tenido conocimiento del intento de suicidio de un compañero cercano en el último año. 


\subsection{Las circunstancias de las acciones autodestructivas y sus implicaciones para la prevención}

El 80,95\% de estudiantes que realizaron un intento de suicidio en el último año pertenecían al sexo femenino y el 19,04\% al masculino. Quienes reportaron haber tenido conocimiento de un suicidio consumado en el último año, el $80 \%$ de los casos era masculino y el 20\% femenino. De quienes reportaron haber conocido de cerca un intento de suicidio, los autores de dichos intentos fueron: $76,47 \%$ de sexo femenino y $23,52 \%$ de sexo femenino. Estos datos concuerdan con los resultados encontrados por la mayoría de los investigadores alrededor del mundo en relación con la distribución de los sexos en cuanto al intento de suicidio y el suicidio consumado, a saber, que por cada hombre que intenta suicidarse lo intentan tres mujeres, y que por cada mujer que consuma el suicidio lo consuman tres hombres.

De este dato se pueden derivar algunas orientaciones para prevenir el suicidio en el escenario universitario, relacionadas con lo que podríamos denominar un enfoque de género, por cuanto muestra que los estudiantes de sexo masculino tienen un riesgo tres veces mayor de suicidarse que sus compañeras de sexo femenino, aunque los intentos fallidos de quitarse la vida son tres veces menos frecuentes que las de ellas. Si a esto se agregan otros elementos relativos a las diferencias de género en nuestra cultura machista, como la mayor licencia para las expresiones afectivas y la mayor disposición a la verbalización de los estados subjetivos por parte de las mujeres, no se deben desvalorizar los indicios de ideaciones o comportamientos suicidas o parasuicidas de los estudiantes varones, lo cual no significa descuidar las precauciones correspondientes en lo referente a los indicios que aportan las estudiantes de sexo femenino.

Las ideaciones suicidas son más frecuentes los fines de semana, mientras que los intentos y los suicidios consumados entre el lunes y el jueves. Respecto a los métodos, la intoxicación y las autolesiones, son más frecuentes en las ideaciones y los intentos fallidos, mientras que el ahorcamiento es más frecuente en los suicidios consumados. El lugar de ocurrencia de los suicidios consumados suele ser la vivienda, y tanto los intentos como los suicidios consumados son más frecuentes durante el período académico. Lo anterior nos enseña que una persona en riesgo suicida no siempre despliegua manifestaciones, acciones o preparativos extraordinarios y altamente notorios. Cuando una ideación suicida está en proceso suele encontrar los medios para su realización en los momentos y lugares más cotidianos, y con los medios más simples 
que la mayoría de las personas tienen a mano. En este sentido, la orientación es no esperar manifestaciones vistosas y dramáticas y comprender que una persona con un plan suicida, puede ser muy discreta.

\subsection{Acontecimientos y acciones que influyeron en los comportamientos autodestructivos}

Sobre los acontecimientos de la infancia relacionados con comportamientos autodestructivos, sobresalen dos: las seducciones, abusos y agresiones sexuales, y las situaciones de maltrato o acoso físico o simbólico por parte de otros significativos.

\subsubsection{Influencia de los acontecimientos de la infancia}

Los estudiantes víctimas de prácticas incestuosas en su infancia suelen mostrar marcas muy graves que los acompañan a lo largo de sus vidas. Las prácticas incestuosas dejan como secuela en las víctimas un sentimiento de no ser dignas de ser valoradas y amadas por otros. Es común que en las expresiones de las víctimas surjan significantes como el sentirse sucias, manchadas o que nunca van a encontrar a nadie que se fije en ellas o que llegue a valorarlas. Es un sentimiento de exclusión del mundo de los intercambios simbólicos en el campo del amor y la sexualidad. Estos procesos suelen estar acompañados de ideas de autodevaluación generalizada que toman la forma de ideas de no valer nada, de ser una especie de desecho. La OMS (2014) conforma la relación entre estos hechos y el suicidio, cuan- do afirma que "los tabúes, el estigma, la vergüenza y la culpa oscurecen los comportamientos suicidas (p. 36). Y argumenta además que los traumas psicológicos que han padecido las personas, así como el abuso, influyen en el estrés emocional pudiendo llevar a las más vulnerables a estos comportamientos suicidas. Johnson et al. (2002) afirman que los jóvenes que en la niñez han sido víctimas de violencia física, maltrato, abuso sexual y otras privaciones afectivas, tienen un mayor riesgo de suicidio. Estos procesos subjetivos pueden permanecer en un estado latente durante la pubertad y la adolescencia, favorecidos por un alejamiento del individuo del mundo de los intercambios amorosos, pero en la juventud, justamente en el período de sus estudios universitarios, un desencuentro amoroso puede desencadenar sus efectos destructivos. Además, como lo plantean Ben-Efraim, Wasserman y Sokolowsky (2013) estos factores adversos ocurridos 
durante la niñez tienen un efecto acumulativo y contribuyen al incremento del riesgo de suicidio.

El desconocimiento sobre la historia personal de los jóvenes estudiantes que intentan suicidarse, o consuman el suicidio, conduce a explicar estos hechos en función de rupturas amorosas, las cuales suelen operar como detonantes, más que como causas eficientes en sí mismas.

Otras violencias físicas y simbólicas de los otros significativos, especialmente los padres y cuidadores en la infancia, también dejan secuelas subjetivas profundas que pueden ser detonadas en el período universitario, con efectos autodestructivos. La subjetividad humana se constituye a partir de la identificación con los otros significativos, y con estas identificaciones se interiorizan también las agresiones, rechazos y devaluaciones de las que fueron objeto en la infancia. No obstante, como lo afirma Carmona (2012): "ni alguien que haya sido víctima de violación y violencias sexuales y físicas en su infancia, puede argumentar que está predestinado a repetir- las como victimario en su vida adulta" (p. 320).

Las consecuencias derivadas para la prevención del suicidio en jóvenes universitarios, relacionadas con las agresiones padecidas en su infancia, quizás sean muy limitadas, pero al menos nos advierten de la fragilidad mayor que presentan los estudiantes cuya historia personal está marcada por esta condición, para brindarles un mayor apoyo en los servicios de bienestar universitario, en casos donde los acontecimientos del presente desencadenen en ellos procesos autodestructivos. Las estudiantes suelen presentar más casos de agresiones sexuales en su historia infantil, mien- tras que los varones reportan más historias de violencias físicas y simbólicas no vinculadas directamente con la sexualidad.

\subsubsection{Los acontecimientos del presente}

Cuando trataban de explicar los comportamientos suicidas sobresalieron los acontecimientos del presente, referidos en mayor proporción a tensiones interpersonales con los otros significativos. Contrario a lo que podía esperarse, no se trataba de grandes eventos relacionados con pérdidas de seres queridos, quiebras económicas, fracasos académicos o laborales, sino situaciones más o menos duraderas en las que se veían rechazados por las personas más cercanas. Riñas, decepciones o tensiones con la familia, la pareja o los amigos, sobresalen en este campo, pero también las situaciones de exclusión en sus interacciones con los compañeros de 
estudio y trabajo, por la condición de pertenecer a una minoría étnica, sexual, social o simplemente por su condición de foráneo. Esta situación, de acuerdo con la OMS (2014) "puede ser continua, endémica y sistémica y puede dar lugar a una continuidad de acontecimientos vitales estresantes, como pérdida de la libertad, rechazo, estigmatización y violencia, que pueden provocar comportamientos suicidas" (p. 36).

Esto pone de relieve la gran fragilidad de nuestros jóvenes y la incidencia de las prácticas de rivalidad hostil, exclusión, segregación, estigmatización y matoneo en los comportamientos autodestructivos de los estudiantes.

No hay que soslayar la influencia de violencias estructurales a nivel social y cultural, ya que los estudiantes de ambos países participantes en la investigación pertenecían a sectores sociales de clase media baja y baja, y cotidianamente debían soportar las violencias simbólicas relacionadas con las injusticias sociales, la falta de oportunidades, las presiones económicas y la ostentación impúdica de los jóvenes de clases altas de sus lujos y su capacidad de derroche, de manera directa o por los diferentes medios de comunicación. Estos factores socioeconómicos como la pobreza y el desempleo, también han sido reportados como factor de riesgo para el suicidio (García, 2015).

Las orientaciones para prevenir estos factores relacionados con los eventos de la vida presente de los estudiantes son muy claras y permiten desplegar más estrategias eficaces para la prevención de los comportamientos autodestructivos.

Respecto a las situaciones de tensión con otros significativos del ámbito familiar y de pareja, los profesionales de los servicios de bienestar, y los tutores u orientadores personales, pueden ser aliados muy significativos para ayudarle a los estudiantes a comprender y transformar estas situaciones problemáticas. Existen instituciones de educación superior con programas de padrinazgo o tutoría, en los cuales algunos estudiantes cualificados de los niveles superiores aportan en el apoyo psicosocial de los estudiantes de los niveles inferiores que requieren de algún acompañamiento especial.

También es muy importante el trabajo de algunas instituciones con los estudiantes que, por sus estudios, han debido dejar sus poblaciones de origen donde viven sus familias y otras redes de apoyo La creación de las colonias regionales, la generación de actividades de integración, las estrategias de padrinazgos, el nombramiento de tutores afectivos y otros recursos similares, serán en un apoyo importante para ayudarles a los estudiantes migrantes a superar las crisis inevitables 
que produce la pérdida de las redes de apoyo. Además, como lo plantean Blandón, Carmona, Zulián y Medina (2015), el ingreso a la universidad es un evento estresante, ya que el joven debe adaptarse a un entorno completamente nuevo, el cual implica asumir cambios significativos en su forma de adquirir conocimientos, además de algunas alteraciones en sus redes de apoyo, y esto podría aumentar el riesgo de que los estudiantes universitarios generen sintomatología clínica. (p. 475).

Sobre las rivalidades hostiles entre los estudiantes, algunas investigaciones muestran cómo en las profesiones socialmente más prestigiosas y asociadas al poder social y económico, y que conllevan mayores niveles de exigencia académica, se presentan tasas más altas de suicidio que en las demás; las facultades de medicina son emblemáticas en una desafortunada paradoja y las siguen las facultades de derecho e ingenierías. Cabrera, Zúñiga y Coria (2011) encontraron en su estudio niveles altos de ideación suicida, con mayor prevalencia en los estudiantes de medicina y biología.

El reto y las oportunidades de prevención de los comportamientos autodestructivos por parte de direcciones de bienestar y los directivos académicos de estas facultades, son muy significativos, y se pueden centrar en generar dinámicas colaborativas de trabajo, en otorgar estímulos académicos a estudiantes más destacados, en estrategias para nivelar a los estudiantes con mayores dificultades académicas. Esto implica romper con la naturalización del individualismo indolente y la competitividad hostil que hacen parte de nuestra cultura.

Con respecto a la influencia cultural, las universidades son por excelencia escenarios Ilamados a liderar los cambios sociales y culturales. Todas las campañas que contribuyan a la crítica a los comportamientos xenófobos, racistas, chovinistas, sexistas y de exclusión o discriminación de minorías, contribuyen a generar condiciones favorables para la afirmación de la vida de los seres humanos en condiciones de vulnerabilidad simbólica por su condición minoritaria. Dicho de otra manera, los movimientos sociales que trabajan por la visibilización de las minorías y la reivindicación de sus derechos, y la afirmación de la diversidad como un valor fundamental de las sociedades democráticas, están en la vía de construir contextos sociales más propicios para la afirmación de la vida y la prevención de los comportamientos autodestructivos.

Cabe aportar una reflexión sobre los resultados de algunas investigaciones de corte epidemiológico sobre el suicidio, basadas en los "factores de riesgo", a partir de correlaciones de factores, según las cuales el pertenecer a ciertos grupos sociales minoritarios o estigmatizados son factores de riesgo en relación con la conducta 
suicida. No es extraño encontrar entre estos grupos el pertenecer a minorías étnicas, a grupos de orientaciones sexuales diferentes de las tradicionales, tribus urbanas con opciones estéticas diferentes a la mayoría, entre otras (Haas et al., 2011). La crítica a la interpretación simplista de la correlación de factores, es que no es en sí misma la orientación sexual o la pertenencia a un grupo étnico o una minoría estética lo que constituye el factor de riesgo, sino las diferentes formas de violencia física y simbólica que ejercen los grupos sociales mayoritarios contra los grupos minoritarios, y que cobra mayor relevancia cuando se acude a las redes sociales. Como lo reporta Brunstein (2010) la ciberintimidación ha cobrado mucha fuerza y es un factor de riesgo para conductas suicidas.

Ignacio Martín Baró acuñó el concepto de “trauma psicosocial” para referirse a las actitudes más o menos generalizadas de grupos humanos sometidos a diferentes formas de violencias, como el fatalismo, la desesperanza, la tendencia a autoexcluirse

y ciertas actitudes de predisposición defensiva, además de comportamientos autodestructivos de los individuos pertenecientes a minorías excluidas, mediante los cuales los individuos de estos grupos humanos interiorizan el rechazo, las actitudes de exclusión y los gestos destructivos de los grupos mayoritarios. Tal es el caso de los refugiados y migrantes, así como las personas que estuvieron o aún permanecen en la cárcel, grupos en los cuales, según la OMS (2014), se ha encontrado relación entre su situación de exclusión y el suicidio.

\subsection{El papel de los vínculos en los comportamientos autodestructivos y en su prevención}

En la investigación mencionada se preguntó a los participantes sobre el papel de los vínculos en los comportamientos autodestructivos. Las respuestas permitieron constatar esa posición bivalente de los vínculos familiares que son a la vez el mayor factor protector y el mayor factor de riesgo de los comportamientos autodestructivos en los niños y en los jóvenes. En el caso concreto de los estudiantes universitarios, la mayoría de los estudiantes coincidieron en atribuir a los vínculos familiares el papel fundamental en las ideaciones, los intentos de suicidio y los suicidios consumados a los que hicieron referencia. Llama la atención que más de la mitad de las respuestas apuntan a la familia como conjunto total o a un grupo plural de integrantes de la familia, por ejemplo "mi mamá y mis dos hermanas", "mis papás y mi hermano" etcétera. Estos hallazgos coinciden con los reportados por otros investigadores 
quienes encontraron relación entre la violencia, la disfunción familiar y el divorcio de los padres con las conductas suicidas (Cañón, et al., 2012; Mouaffak, Gallegos, Stark, Linan, et al, 2013; Pinzón-Amado, 2013; Sánchez \& Robles, 2014; Marchand, Castaigne, et al., 2015). Pero, más que la presencia de conflictos familiares es su inadecuado manejo el que los convierte en factor de riesgo (Sánchez, Guzmán y Cáceres, 2005; Pérez et al., 2007; Sarmiento y Aguilar, 2011).

Los estudiosos de los fenómenos grupales muestran que en grupos pequeños como la familia se instalan dinámicas colectivas que pueden llegar a ser altamente destructivas para alguno o algunos de sus integrantes. Enrique Pichón Riviere acuñó la categoría denominada el "chivo emisario", para denominar ese integrante al que el grupo le asigna el rol de ocuparse de la dimensión sintomática de la que no quieren hacerse cargo los demás integrantes del grupo. Según el autor, los grupos humanos más saludables y más vitales son aquellos en los que la dimensión patológica del grupo (lo sintomático, lo anómalo, la locura) se distribuye de una manera, por decirlo así, más democrática entre los integrantes del grupo; y los grupos humanos más enfermizos y que potencian la pulsión de muerte en sus integrantes, los que endosan la dimensión patológica del grupo a alguno o algunos de sus integrantes quienes desempeñan el rol del chivo emisario.

Nos estamos refiriendo a tensiones o dificultades de nuestros estudiantes con uno u otro de los integrantes de la familia, por ejemplo, el padre, la madre o un hermano, sino de una dinámica colectiva en la que participan dos o más integrantes. Se trata de fenómenos muy cotidianos, pero algunos casos tienen consecuencias catastróficas, como cuando uno de los hijos es el paradigma del éxito y otro del fracaso, o uno de ellos es el símbolo de la virtud y otro del vicio, uno de la cordura y otro de la desmesura, etc., No es casualidad que aquellos integrantes, a los que el grupo familiar les asigna el rol del chivo emisario, desarrollen comportamientos autodestructivos asociados con dicho rol, dentro de los cuales pueden incluirse el abuso de sustancias psicoactivas, comportamientos de transgresión de la ley, negligencias en el autocuidado, acciones temerarias, etcétera. Estos comportamientos autodestructivos, originados por el lugar perverso que les ha asignado el grupo familiar, terminan convirtiéndose en la pseudo-explicación que la misma familia utiliza para estigmatizar y aislar más a dicho integrante, con lo cual se produce lo que Edgar Morin llama un fenómeno de "causalidad recursiva" en el cual la consecuencia termina reforzando la causa en una lógica destructiva espiralada. 
Pero, así como la familia puede ser un factor de riesgo, también funciona como un factor de protección frente a las conductas autodestructivas, como lo reportan varios autores (Oliva, 2006; Andrade, 2012; Monge, Cubillas, Román \& Abril, 2007) quienes encontraron en sus investigaciones que los padres afectuosos, que establecen una buena comunicación con sus hijos y los animan a ser autónomos, son un factor protector para sus hijos.

El "matoneo" es ciertamente la denominación contemporánea de una de las prácticas típicas de acoso y violencia física y simbólica en el escena- rio académico. Más allá de la importancia de los esfuerzos por identificar los líderes o los responsables más directos del fenómeno para efectos disciplinarios o legales, algunas investigaciones estudian la responsabilidad colectiva en el fenómeno, y muestran el papel de diferentes grupos, como los observadores que festejan y los líderes que contribuyen con su silencio cómplice. Algunos investigadores del bullying han acuñado el neologismo "bullicidio", para subrayar el papel que tiene el bullying como causa específica en algunos casos de suicidio.

Podríamos decir que el "chivo emisario" en el contexto familiar y el "bullying", como síntoma colectivo, y ambos como fenómenos universales (como lo es también el suicidio), son una muestra de la potencia suicidógena de las interacciones colectivas de los seres humanos. Esto sugiere que en la teorización sobre las interacciones se mantenga siempre presen- te esa condición de moneda de dos caras señalada más arriba, en virtud de la cual los contextos vinculares son, a la vez, un poderoso factor protector frente a las tendencias autodestructivas y un potente factor de riesgo en la medida en que puede potenciarlas hasta límites mortales.

Ahora señalemos algunas dinámicas sociales que funcionan de acuerdo con la misma lógica y donde se pueden observar el factor suicidógeno de los grupos sociales.

En realidad se trata del mismo principio del chivo emisario, operando en el contexto más amplio de la vida social, que toca con lo político. Se podría decir, con Pichon Riviere, que en una sociedad saludable los bienes físicos y simbólicos están distribuidos de una manera más o menos equitativa entre sus integrantes, y que en una sociedad enferma la distribución de los bienes físicos y simbólicos es muy inequitativa.

Una sociedad enferma genera grandes grupos humanos articulados en torno a síntomas colectivos: los drogadictos, las prostitutas, los delincuentes, los habitantes de calle, los narcotraficantes, los guerrilleros, entre muchos otros. Cuando las pseudo- 
explicaciones que confunden el efecto con la causa se reifican, se genera una visión de la sociedad según la cual la drogadicción es responsabilidad de los drogadictos, la prostitución de las prostitutas, la delincuencia de los delincuentes y la guerrilla de los guerrilleros. Se produce la ficción de una sociedad de seres fundamentalmente virtuosos, pacíficos y amantes de la ley, que no son totalmente felices por culpa de los viciosos, los ilegales y los violentos. Esta visión de la sociedad deriva en políticas que radicalizan la exclusión de estos grupos y que con frecuencia se deslizan hacia propuestas de exterminio (Ilamadas eufemísticamente de "limpieza social”) que son congruentes con dicha visión. Insistimos que no es casual que las tasas de suicidio sean más altas en los grupos minoritarios y segregados, que encarnan los síntomas sociales, que en los individuos pertenecientes a los grupos mayoritarios, aceptados e integrados en ellos.

Sobre las consecuencias para la prevención, podemos decir que los profesores universitarios pueden y deben aprovechar la investidura de su rol, para favorecer dinámicas de solidaridad, cooperación e inclusión en las aulas; también en fomentar el reconocimiento de la diversidad como un valor y, donde sea posible, convertirse en un apoyo temporal para estudiantes en situaciones de exclusión por parte de sus compañeros.

A las direcciones de bienestar universitario, con el apoyo de las facultades de ciencias sociales y humanas, les corresponde la tarea de generar estrategias, programas y proyectos para contrarrestar las diferentes formas del bullying, que actualmente son favorecidas por el anonimato que posibilitan las nuevas tecnologías en las universidades.

La universidad es, por excelencia, el escenario social donde, con el apoyo de investigaciones y debates científicos, es posible contribuir a neutralizar las ideologías que favorecen la producción de síntomas sociales, para después usarlos como pseudoexplicación de los males de la sociedad y dirigir contra ellos la destructividad de la sociedad.

\subsection{El papel de las significaciones en los comportamientos autodestructivos y su prevención}

Se presentan a continuación la discusión y las conclusiones para la prevención derivada de las significaciones mencionadas en las narrativas de los estudiantes de ambos países, relacionadas con los comportamientos autodestructivos. Tal como se ha 
mencionado en otros capítulos de este libro, las significaciones están profundamente anudadas con las acciones y las interacciones, por lo cual en algunos casos será inevitable la sensación de volver con otro matiz sobre un tema ya tratado. De todos modos se considera importante el esfuerzo, por la posibilidad de influir directamente en las significaciones de manera independiente y, por medio de ellas, en las acciones y las interacciones.

La significación más destacada en las narrativas de los estudiantes en relación con las ideaciones suicidas, los intentos de suicidio y los suicidios consumados, se refiere al "abandono, la soledad y el desamor", como lo reporta Carmona (2012) "el aislamiento y el abandono también son factores de vulnerabilidad frente a las distintas formas de empuje social al suicidio y las tendencias autodestructivas de la propia persona (p. 331). Esto permite corroborar una constatación repetida en el campo de la psicología y la psicología social, a saber, que los seres humanos para sobrevivir necesitamos tanto del afecto como el cuerpo de los nutrientes.

Estos jóvenes corroboran el hallazgo realizado por el psicoanalista inglés René Spitz, quien acuñó el término "síndrome del hospitalismo" para referirse a la causa de la muerte de niños entre los seis y los 18 meses, internados en una institución a raíz del fallecimiento de sus madres. El valor teórico de esta categoría reside en la observación aguda del autor quien constató que de un grupo de niños que recibían los mismos cuidados, en términos de nutrición y otras necesidades básicas, muchos de ellos morían, y quienes sobrevivían y se aferraban a la vida recibían algún tipo de expresión afectiva por los profesionales, o incluso por los empleados de servicios varios, quienes les dedicaban momentos para arrullarlos o manifestarles afecto. Con sus narrativas, los estudiantes universitarios participantes en la investigación señalan que la deprivación afectiva para una persona joven es suicidógena, y de paso nos brindan pistas para la prevención del fenómeno.

El reto para los equipos académicos y los profesionales de los servicios de bienestar de las universidades, derivado de esta constatación, tiene varios frentes. La estrategia general se orientaría en función contribuir a mantener las mejores condiciones afectivas posibles para la afirmación de los estudiantes en su deseo de vivir y hacerse profesionales.

Algunas de las acciones estarían orientadas a lo que podríamos llamar la sensibilización y orientación de sus familias por medio de reuniones, cartas colectivas, boletines y otro tipo de comunicaciones para que comprendan la importancia de dejarle sentir a los estudiantes el afecto positivo mediante manifestaciones claras. Esto 
puede ser mucho más significativo para los estudiantes provenientes de otras regiones, más expuestos al sentimiento de soledad y abandono que quienes viven en la ciudad. Nuevamente aparece la necesidad de generar dinámicas que fomenten la integración de los estudiantes y la creación de agrupaciones, asociaciones y espacios colectivos de apoyo mutuo. Habrá casos en los cuales, de acuerdo con el diagnóstico, corresponda realizar estrategias de alfabetización emocional.

Un segundo grupo de narrativas se agrupó en la categoría denominada "alivio, liberación o solución radical”. Se trata de una significación radicalmente distinta de la anterior. En este caso, las significaciones asociadas a los comportamientos autodestructivos no se refieren a la tristeza profunda, por la ausencia de los otros, sino del agobio y el sentimiento de atrapamiento por el sentimiento de acoso y acorralamiento. El comportamiento autodestructivo aparece como un escape a una especie de hiperpresencia del otro, que no recibe afecto tierno sino el acoso hostil. No es casual que en algunos casos se asocie este sentimiento con los períodos de exámenes, propio del mundo académico. Además de la adecuada planeación de las asignaciones académicas, y su razonabilidad en función del número de créditos de cada curso, puede ser importante desplegar diferentes estrategias para generar conciencia en los estudiantes trabajadores sobre la importancia de no tomar más materias de la que puede cursar con responsabilidad. La desmesura en la planeación académica puede funcionar al servicio de las tendencias autodestructivas. En estos casos, expresiones del argot popular como "matarse estudiando", "quemarse las pestañas" etc., adquieren todo su sentido y han sido reportados también como factores de riesgo para el suicidio (Hernández et al., 2015). Queda también el reto para los servicios de bienestar, con el apoyo de comunicadores y educadores, de fomentar una relación con la formación profesional y la vida académica en general más vinculada con la pasión y con el deseo, que con la obligación, la competencia y la presión.

Una tercera significación se agrupó bajo la categoría de "respuesta frente a una decepción radical". Se trata de una significación bien diferenciada de las dos anteriores. En este caso se trata de una respuesta agresiva ante la caída de un ideal, que se vuelve contra la propia persona. La idealización suele tener como contracara un componente fuerte de hostilidad ya listo para volverse contra el objeto y contra la propia persona cuando el objeto idealizado no se mantiene a la altura de lo que el sujeto espera de él. Acá es poco lo que se puede hacer en términos de prevención, porque el fenómeno de la idealización está relacionado con dinámicas inconscientes y procesos afectivos, que difícilmente se pueden afectar desde el mundo de los argumentos, pero todo lo que se puede hacer en el mundo académico por fortalecer el 
pensamiento crítico en nuestros estudiantes contribuye a ayudarles ser menos susceptibles a construir idealizaciones, y al dolor subjetivo que provoca la caída de los ideales. Igualmente, requieren orientación para comprender sus estados emocionales y reparar las emociones negativas, así como herramientas para afrontar los problemas, situaciones que Suárez, Restrepo y Caballero (2016) encontraron relacionadas con la ideación suicida.

Una cuarta significación fue agrupada bajo la categoría de "ideas autodenigrantes o de autodevaluación". La baja autoestima ha sido reportada por varios investigadores como factor de riesgo para el suicidio (Mouaffak, Marchand, Castaigne, et al., 2015; Gallegos, Stark, Linan, et al., 2013; Cañón, et al., 2012; Pinzón-Amado A., 2013; Sánchez \& Robles, 2014). En general, estas ideas no suelen tener un fundamento racional y responden a la interiorización de agresiones de otros significativos, que fueron externas y que luego se repiten interiormente bajo la forma de autoagresiones. En los casos extremos estos casos derivan en la melancolía, una de las formas más graves de la psicosis. Para la intervención de este tipo de significaciones, la psicoterapia individual es lo más indicado. Cuando un profesor, un estudiante o un profesional del servicio de bienestar universitario, encuentre un estudiante en riesgo suicida, donde las ideas autodenigrantes o de autodevaluación sean el rasgo predominante, la indicación más razonable es proporcionarle ayuda profesional.

Una quinta significación agrupada bajo la categoría de "falta de sentido de la vida" está muy ligada con la depresión en el sentido técnico de la palabra (no en el sentido de tristeza, empleado en el argot popular). La falta de sentido da cuenta de un agotamiento del deseo, o más exactamente de la energía de la pulsión de vida, ocasionado por algún conflicto profundo. En estos casos, encontrarle ayuda profesional es lo más indicado. La depresión es uno de los principales factores relacionados con el suicidio (Rodríguez et al., 2012; Sánchez et al., 2010; Castrillón et al., 2016).

Finalmente, hay otro grupo de narrativas en las que no aparece ninguna significación sino la idea de quitarse la vida, sin una representación que justifique este impulso. Estos casos son los más graves desde el punto de vista psicológico, y los que requieren con mayor urgencia de una atención psicológica, porque en la medida en que no estén asociados con ningún proceso ideativo y aparezcan como acto puro, conllevan un mayor riesgo para el estudiante. 


\subsection{Acciones que ayudaron a los estudiantes a evitar el suicidio y a afirmarse en la vida}

De las respuestas aportadas por los estudiantes sobrevivientes de los comportamientos autodestructivos a la pregunta por las acciones que evitaron el suicidio y les ayudaron a seguir viviendo, analizadas en los tres capítulos anteriores, se puede concluir que el grupo más representativo, en términos porcentuales, presenta algunos elementos en común; el primero, se trata de acciones que hacen posible la elaboración simbólica en la medida que involucran procesos de verbalización y reflexión. Otro elemento sobresaliente y vinculado al anterior se relaciona con el efecto catártico o de alivio que propicia la verbalización en el contexto de una sesión terapéutica, la conversación con un religioso con capacidad de es- cucha, o con un amigo. Como lo plantean Carmona y Cañón:

A veces, por la particularidad de la situación, una compañía cualificada, mientras la persona supera la crisis o se logra conseguir algún tipo de ayuda, puede ser de alto valor. No siempre la persona en riesgo de suicidio es capaz o está dispuesta a hablar. Y no siempre el docente tiene la capacidad, o está en condiciones de escuchar lo que el estudiante tiene para decir. Los forzamientos en estos casos pueden entorpecer la intención de ayudar. La capacidad de discernir lo posible y lo oportuno son fundamentales en todos los eventos humanos, y particularmente en el riesgo suicida. A veces una compañía solidaria, respetuosa, amable puede ser lo más indicado. (p. 116).

Otro elemento notorio de las acciones es construir nuevos vínculos o fortalecer los existentes. En algunas de las acciones se involucra el cuerpo por la vía de la lúdica y la danza o de las actividades manuales, y en otras presente, el adquirir nuevos conocimientos relacionados con sus campos de interés. Finalmente, en algunas acciones sobresale la relación con lo religioso.

El segundo grupo de respuestas más representativas en términos porcentuales, sobre acciones que contribuyeron a la afirmación de la vida, se sitúan las relacionadas con el fortalecimiento de vínculos familiares, de pareja y de amistad. En estas respuestas los estudiantes nos brindan claves importantes para las estrategias de intervención de los profesionales de los servicios de bienestar: todo lo que se pueda 
hacer para ayudarles a reparar y fortalecer los vínculos con los otros significativos contribuirán de una manera efectiva a su afirmación en el mundo. "El elemento afectivo de los vínculos humanos tiene la doble condición de factor de riesgo y protección frente a los comportamientos autodestructivos" (Carmona y Cañón, 2016, p. 123).

\subsection{Vínculos y significaciones que contribuyen a la evitación del suicidio y a la afirmación de la vida}

En cuanto los vínculos, la familia como conjunto, la pareja y las relaciones de amistad, aparecen en primer plano, por lo cual los esfuerzos más importantes en las intervenciones se deben orientar hacia ellos.

Las significaciones protectoras también recaen mayoritariamente en estos campos de la familia, la pareja y las relaciones de amistad, y aparece un elemento digno de una consideración especial, relacionado con el papel del pensamiento crítico en la detención de un proceso autodestructivo y la afirmación en el deseo de vivir. Este elemento de debate interno nos puede dar una importante pista para la prevención y la intervención, porque nos anuncia que en los estudiantes en riesgo de suicidio existe un debate interno, y que si se logran situar con claridad los argumentos a favor de la afirmación de la vida se puede ser mucho más eficaz en la tarea de apoyo, que cuando no se cuenta con ellos y se pretende intervenir desde los propios argumentos quien brinda la ayuda. La visión de la subjetividad, como una estructura de autointeracciones, y del pensamiento como un diálogo o discusión interna, es fundamental para operacionalizar esta conclusión que fruto de los resultados de la investigación, para la prevención e intervención de los comportamientos autodestructivos en estudiantes universitarios.

Otro apoyo importante, para ayudarles a los estudiantes en riesgo a afirmarse en el mundo, lo puede proporcionar el profesional de los servicios de bienestar en las representaciones relacionadas con las expectativas y proyectos futuros. 


\subsection{El papel de la universidad en la afirmación de la vida de los estudiantes}

Una de las conclusiones fundamentales de esta investigación es que el mundo universitario posee todas las condiciones para ser un espacio por excelencia preventivo de los comportamientos autodestructivos, y potenciador de la afirmación del deseo de vivir. Esto es un importante punto de referencia para que los directivos y profesionales de bienestar universitario analicen bien lo que ocurre con las acciones, interacciones y significaciones de las unidades académicas donde empiecen a manifestarse de una manera significativa comportamientos autodestructivos en los estudiantes. Mencionemos las conclusiones relacionadas con las potencias de las instituciones universitarias al servicio de la afirmación de la vida.

Respecto a las acciones, un elemento característico del mundo de la educación superior es la riqueza y la vitalidad de las actividades que ofrece a sus estudiantes, por más modesta que sea la institución. El hecho mismo de que la mayoría de las actividades se centren en la investigación y el aprendizaje, contribuye al enriquecimiento y flexibilización del universo simbólico del estudiante y a la neutralización de las ideas relacionadas con los procesos autodestructivos. La universidad es por excelencia un espacio de autotransformación para los estudiantes, que moviliza de manera positiva sus esquemas mentales en función de procesos vitales.

Sobre las interacciones, las instituciones de educación superior son escenarios privilegiados para que los estudiantes construyan nuevos vínculos con otras personas con quienes comparten afinidades electivas. Quizás no sea gratuito que algunos de los vínculos significativos que acompañan a las personas en su vida profesional se construyen justamente en las aulas universitarias. Las mismas dinámicas de la vida académica generan las condiciones para que los vínculos que allí se construyen sean más maduros y cualificados que los dados en otros contextos sociales. El aporte del mundo de la educación superior a la calidad de los vínculos entre los seres humanos en una sociedad es algo que quizás no ha sido suficientemente apreciado. Y respecto al problema de los comportamientos autodestructivos, reiteramos que la calidad de los vínculos de un ser humano contribuye de una manera fundamental a su afirmación en el mundo, mientras que los vínculos altamente inestables y conflictivos favorecen el riesgo de los comportamientos autodestructivos. 
Respecto a las significaciones, el elemento más potente de la vida universitaria es la dimensión teleológica o prospectiva que significa para la mayoría de los estudiantes una carrera universitaria. Esta significación, ligada a un futuro posible y deseable, repercute directamente en la significación de sí mismos, como obras autopoiéticas en proceso de construcción, lo cual tiene mucha importancia en función de la afirmación de la vida.

Finalmente, las universidades poseen recursos invaluables para generar estrategias de prevención de comportamientos autodestructivos en sus estudiantes, y para promover en ellos la afirmación de la vida.

Si las estrategias se orientan hacia las significaciones, la comunicación y la educación serán disciplinas imprescindibles en la construcción de boletines cuidadosamente elaborados, tanto desde el punto de vista pedagógico como en cuanto a los criterios de calidad de la publicidad social.

Estos resultados pueden ser la base para construir, a partir de cada uno de ellos, una unidad o una pieza comunicativa orientada no solamente a la prevención de los eventos negativos, sino hacia la promoción de estrategias de afirmación de la vida.

Las facilidades que brindan las nuevas tecnologías permiten que las unidades de bienestar de las universidades puedan manejar un canal di- recto de comunicación, por medio de correos electrónicos, con los padres y cónyuges de los estudiantes, y desarrollar con ellos campañas de orientación para el fortalecimiento y cualificación de los vínculos, y la prevención de comportamientos autodestructivos. Como lo plantean Collin et al. (2011) las redes sociales tienen un papel fundamental como estrategia para contribuir a la prevención del suicidio.

Estas estrategias masivas no excluyen la posibilidad de generar otros espacios de interacción más directos con los otros significativos de los estudiantes, como son las reuniones y talleres con padres y parejas, que se pueden realizar al menos una vez por semestre, para convertirlos en auténticos aliados de la permanencia con calidad del estudiantado en sus programas de formación, la prevención de comportamientos autodestructivos y la cualificación de los vínculos. En estas reuniones se puede reservar un espacio específico para orientar acerca de estrategias de manejo e identificar dificultades en la comunicación relacional y, de ser necesario, facilitar referidos para intervención psicosocial.

Los elementos abordados en este apartado también proporcionan importantes herramientas a los equipos psicosociales de las unidades de bienestar universitario 
para generar piezas informativas destinadas a los mismos estudiantes, de manera que éstos aprendan a identificar las significaciones, los vínculos y los acontecimientos ligados a los comportamientos autodestructivos, y puedan solicitar ayuda oportuna a los equipos psicosociales de tales unidades. Integrar grupos de pares al trabajo de prevención temprana, cuando ello sea preciso, y orientar a los compañeros en riesgo para que busquen ayuda oportuna.

Los capítulos de resultados de esta investigación son, en sí mismos, una herramienta de prevención para los profesionales del campo de la salud mental, de las unidades de bienestar y servicios psicosociales al estudiantado de las universidades, por cuanto les aportan las narrativas típicas de estudiantes con ideaciones suicidas o han realizado intentos de suicidio, lo que, a su vez, les servirá para agudizar la mirada y detectar mejor, de manera oportuna, estudiantes en riesgo de estos comportamientos autodestructivos. Asimismo, propiciar la escucha de la ideación y del intento no sólo como indicador de riesgo sino como signo y herramienta de resistencia, que requiere apalabrar y por lo mismo transformarlas en oportunidades de prevención.

Como herramienta complementaria a este libro se puede construir un claro protocolo de alertas como guía de consulta rápida, accesible tanto para los profesionales como para profesores, empleados de los niveles administrativo y de dirección de cada institución. De hecho, y como producto de la investigación, el equipo investigativo de Puerto Rico diseñó una Guía de Prevención de Suicidio (Maldonado Santiago, Rivera Lugo, Carmona Parra y Jaramillo Estrada, 2015). La guía ofrece respuestas a preguntas como: ¿qué se puede hacer si un estudiante se ha suicidado?, ¿qué podemos hacer si un estudiante ha intentado suicidarse? y ¿qué podemos hacer si un estudiante tiene o ha tenido ideaciones suicidas? Incluye, además, herramientas útiles a la comunidad universitaria, para intervenir efectivamente en estos tres eventos cada vez más vez en nuestras instituciones universitarias.

\section{Referencias}

Andrade, J. (2012). Aspectos psicosociales del comportamiento suicida en adolescentes. Revista electrónica de Psicología Iztacala, 15(2), 688-721.

Ben-Efraim YJ, Wasserman D, Wasserman J, Sokolowski M. (2013). Family-based study of HTR2A in suicide attempts: observed gene, gene $\times$ environment and parentof-origin associations. Mol Psychiatry,18(7):758-66. doi: 10.1038/mp.2012.86 
Blandón, O. M., Carmona, J. A., Mendoza, M. Z. \& Medina, O. A. (2015). Ideación suicida y factores asociados en jóvenes universitarios de la ciudad de Medellín. Revista Archivo Médico de Camagüey, 19(5), 469-478.

Brunstein Klomek A, Sourander A, Gould M. (2010). The association of suicide and bullying in childhood to young adulthood: a review of cross-sectional and longitudinal research findings. Can J Psychiatry, 55(5):282-8.

Cabrera, N., Zuñiga, M. \& Coria, A. (2011). Depresión e ideación suicida en estudiantes de la FESI: Un estudio piloto. Revista electrónica de Psicología Iztacala, 14(4), 341354.

Cañón S., Atehortúa B., Castaño J. et al. (2012). Factor de riesgo para suicidio según dos cuestionarios y factores asociados en población estudiantil de la Universidad de Manizales. Psicología des de el Caribe, 29(3), 632-664.

Carmona, J. A. y Cañón, S. C. (2016). La prevención del suicidio en el escenario universitario. En: J. A., Carmona (Ed.) Ensayos académicos en torno al suicidio. (pp. 101-124). Cali: Fundación Participar IPS.

Castrillón, J. J., Cañón, S. C., Betancur, M. B., Sánchez, P. L., González, J. G., Rivas, A. G. \& Calderón, C. L. (2016). Factor de riesgo suicida según dos cuestionarios, y factores asociados en estudiantes de la universidad nacional de Colombia sede Manizales. Diversitas, 11(2), 195-205.

Collin, P, J., Metcalf, A. T., Stephens-Reicher, J. C., Blanchard, M. E., Herrman, H. E., Rahilly, K. \& Burns, J. M. (2011). ReachOut.com: The role of an online service for promoting help-seeking in young people. Journal Advances in Mental Health. Promotion, Prevention and Early Intervention, 10(Issue 1), 39-51.

Gallegos, J., Stark, K., Linan-Thompson, S. et al. (2013). Preventing childhood anxiety and depression: Testing the effectiveness of a school-based program in México. Psicología Educativa, 19, 37-44

García Peña, J. J. (2015). El enfoque psicosocial del suicidio. En J. A., Carmona (comp.), el suicidio: cuatro perspectivas (pp. 33-47). Medellín: Fondo Editorial Funlam.

Haas, A. P., Eliason, M., Mays, V. M., Mathy, R. M., Cochran, S. D., D’augelli, A. R., ... Clayton, P. J. (2011). Suicide and Suicide Risk in Lesbian, Gay, Bisexual, and Transgender Populations: Review and Recommendations. Journal of Homosexuality, 58(1), 10-51. http://doi.org/10.1080/00918369.2011.534038 
Hernández, M., De Los Reyes, A., González, Y. y Prats, J. (2015). Caracterización de los adolescentes comisores del intento suicida del Consejo Popular Siboney. Octubre de 2010 a Marzo de 2011. Granma, 19(3), 1-10.

Maldonado S. N., Rivera Lugo, C., Carmona Parra. J. y Jaramillo Estrada, J.C. (2015). Guía para la Prevención del Suicidio en Jóvenes Universitarios: ¿Qué podemos hacer en casos de suicidio, intentos de suicidio e ideación suicida en nuestros estudiantes? Puerto Rico: Pontificia Universidad Católica de Puerto Rico.

Monge, J. A., Cubillas, M.J., Román, R. \& Abril, V. E. (2007). Intentos de suicidio en adolescentes de educación media superior y su relación con la familia. Psicología y Salud, 17(1), 45-51.

Mouaffak, F., Marchand, A., Castaigne, E. et al. (2015). OSTA program: A French Follow up Intervention Program for Suicide Prevention. Psychiatry Research, 230, 913918

Oliva, A. (2006). Relaciones familiares y desarrollo adolescente. Anuario de Psicología, 37(3), 209-223.

Organización Mundial de la Salud (2014). Prevención del Suicidio: Un imperativo Global. Washington, DC. Disponible en: www.who.int.int/mental_health/media/ media_spanish.pdf.

Pérez, I. (2007). Caracterización Psiquiátrica y social del intento suicida atendido en una clínica infantil, 2003-2005. Revista de Salud Pública, 9 (2), 230-240.

Pinzón-Amado, A., Guerrero, S., Moreno, K. et al. (2013). Ideación suicida en estudiantes de medicina: prevalencia y factores asociados. Rev Colomb Psiquiat., 43(S1), 47-55.

Rodríguez, C., José, M., Román Pérez, R., Abril Valdez, E., Barreras, G. \& Lourdes, A. (2012). Depresión y comportamiento suicida en estudiantes de educación media superior en Sonora. Salud mental, 35(1), 45-50.

Sánchez, J. C., Villareal, M. E., Musitu, G. y Martínez, B. (2010). Ideación suicida en adolescentes: un análisis psicosocial. Intervención Psicosocial, 19(3), 279-287

Sánchez, D. y Robles, M. (2014). Factores protectores que promueven la resiliencia ante el suicidio en adolescentes y jóvenes. Pap Psicol, 35, 181-192 
Sánchez, R., Guzmán, Y., Cáceres, H. (2005). Estudio de la imitación como factor de riesgo para ideación suicida en estudiantes universitarios adolescentes. Revista Colombiana de Psiquiatría, 34 (1), 12-25.

Sarmiento, S. y Aguilar, J. (2011). Predictores familiares y personales de la ideación suicida en adolescentes. Revista Psicología y Salud, 21(1), 25-30.

Suárez, Y. P., Restrepo, D. E. y Caballero, C. C. (2016). Ideación suicida y su relación con la inteligencia emocional en universitarios colombianos. Rev. Univ. Ind. Santander Salud, 48(4), 1-16. 